\title{
Montréal et São Paulo dans la dynamique narrative de Monique Proulx et Luiz Ruffato
}

\section{Montreal and São Paulo in the dynamic narrative of Monique Proulx and Luis Ruffato}

Licia Soares de Souza ${ }^{1}$

Submetido em 25 de dezembro de 2016 e aprovado em 26 de fevereiro de 2017.

Résumé: Cette communication consistera en une comparaison de deux ouvrages des Amériques, Ce qu'il reste de moi (2015), de Monique Proulx et Tant et tant de chevaux (2002), de Luiz Ruffato. Dans le premier, le Montréal du XXIe siècle reste le personnage principal, comme le témoin du destin collectif d'êtres intenses avec des voix multiples qui seraient venues concrétiser le rêve de la «Folle Entreprise» de Jeanne Mance. Des héros migrants méconnus deviennent en effet des conteurs, chargés de maintenir et de transmettre les legs personnels et collectifs dans un espace charnière comme la métropole québécoise. Dans le récit brésilien, le personnage principal comme espace-charnière reste la mégalopole de São Paulo. Ruffato présente 69 micro-récits autonomes et hybrides qui rendent compte de l'errance des laissés-pour-compte, des riches et des gens de la vie ordinaire; Noirs, Indiens, Nordestins, pauvres, des personnages de toutes origines et conditions sociales. Les deux romans s'inscrivent dans la littérature actuelle où les interrogations sur la place des individus dispersés entraînent une remise en cause de grands récits et d'identités trop définies.

Mots-clefs: Folle Entreprise. Monique Proulx. Immigrants. Luis Ruffato. São Paulo. Montréal.

\begin{abstract}
This paper compares two works from the Americas: Ce qu'il reste de moi (2015) by Monique Proulx and Tant et tant de chevaux (2002) by Luiz Ruffato. In the first work, Montreal of the XXI century is the main character as the witness of the collective destiny of intense people. Multiples voices from these people would come to realize the Jeanne Mance's dream of The Crazy Enterprise. Unknowns, migrants, heroes, become storytellers. They transmit personnel and collective legacies of a hinged space known as the Metropolis of Quebec. In the Brazilian narrative, the main character is the megalopolis of São Paulo, also a hinged space. Ruffato presents 69 autonomous micro-narratives that show the profusion of poor and rich people that wander in the city. The two novels represent contemporary literature. Questions arise about the place of dispersed people and about the legitimacy regarding big narratives with ideas about defined identities.
\end{abstract}

Key-words: Crazy Enterprise. Monique Proulx. Immigrants. Luis Ruffato. São Paulo. Montreal. 
L'examen des figurations littéraires et cinématographiques des «espaces en devenir» s'est trouvé au cœur du colloque Figures littéraires des espaces en devenir au Québec et au Brésil (XXème $-X X I^{\text {ème }}$ siècles): conflits, traumatismes, interconnexions dans le cadre du $84{ }^{\text {ème }}$ Congrès de l'ACFAS. Inspirée, entre autres, des travaux de Patrick Imbert (2004), la perspective adoptée nous a amenés à prendre en considération la dynamique du changement spatial et du dépassement des frontières rigides. Nous nous sommes demandés: Quels sont les enjeux esthétiques et théoriques d'un tel choix de figuration? Quelles images des espaces en devenir seraient mises en scène dans ces productions?

Nous avons commencé par le rappel d'une réflexion de Pierre Ouellet (2003), «la ville est parlée, bien sûr, contée et racontée, mais elle parle aussi, dans sa langue à elle». Bertrand Gervais (2008) demande: «À quoi ressemblent les villes que nous avons en tête quand nous pensons à ces lieux que nous vivons ou visitons?». Est-ce que les villes pensées sont faites de lignes droites et continues, d'espaces ordonnés et de lieux aisément repérables? Il semblerait qu'en tant que subjectivité énonçante, aussi bien qu'en tant qu'objet énoncé, comme nous en avons tellement débattu, une ville devient déconcertante. Elle apparaît comme l'expression de la nature des tensions conflictuelles qui gouvernent une sémiosphère.

Nous avons maintes fois répété que, dans l'univers culturel désigné par la notion de sémiosphère, il n'est plus question de systèmes de signes dans leurs structures dites immanentes ${ }^{2}$. La ville devient un lieu d'écriture, mais il s'agit d'un lieu qui permet l'affleurement d'espaces déterminés, susceptibles de signifier la complexe diversité qui caractérise une métropole moderne. On admet, dans un premier temps, que la ville est un espace physique et palpable avec ses limites géographiques. La tension entre fragmentation et unité urbaine impose une vision privilégiée des espaces urbains dans lesquels s'entrecroisent des foyers culturels spécifiques. De ce fait, le discours fictionnel rend compte d'un territoire citadin capable de mettre en relation des lieux variés de formations culturelles, de telle sorte que la perspective géographique, qui ne négligera pas ici les enchâssements des thèmes identitaires, sera assortie d'une confrontation entre certaines figures spatiales de la ville, qui la modèlent exactement comme espace en devenir: il y a là une prise en considération de la dynamique du changement spatial et du dépassement des frontières rigides. 
Nous avons envisagé ici la confrontation entre certaines figures spatiales de Montréal, comme celle de l'espace-charnière et celle de l'espace-gigogne ${ }^{3}$. Nous remarquons que ces deux figures spatiales, bien que distinctes, mettent en lumière les rapports complexes que les contextes identitaires locaux et les acteurs sociaux en marge du centre sémiosphérique peuvent entretenir avec la dynamique du réseautage. Nous parlerons davantage de la configuration de ces deux figures au fil de l'analyse des deux ouvrages que nous avons retenus pour cette communication.

\section{Ce qu'il reste de moi}

Dans Ce qu'il reste de moi, la romancière et scénariste Monique Proulx entend montrer plusieurs visages de Montréal comme espace urbain particulier du Nouveau Monde. Pour ce faire, elle crée le personnage Laurel qui - fasciné par les récits de Jeanne Mance au sujet de son projet de la «Folle Entreprise» entre 1642 et 1665 -, décide d'écrire un autre récit sur le Montréal contemporain qui pourrait être un reflet de ce projet du temps de la Nouvelle France. Celui-ci repose sur un rêve dont l'objet est le Nouveau Continent: avec le soutien de grands donateurs privés, ils créeraient une ville «mixte», où les Européens immigrés et les Amérindiens devenus chrétiens vivraient ensemble, dans un climat d'harmonie et de solidarité. Proulx déclare qu'un tel projet de cohabitation harmonieuse n'a jamais existé, bien entendu, mais dans l'esprit des Montréalais, il a nourri pendant des siècles une figuration relative à la construction d'une cité dans laquelle les nouveaux habitants - des immigrants venus des quatre coins du monde -, trouveraient un espace idéal d'accueil pour vivre en communion avec les natifs. La ville se manifeste ainsi comme un écran sémiosphérique qui permet l'affleurement d'un faisceau de relations euphoriques puisées dans un hypotexte historique qui fonctionne lui-même comme texte fondateur ${ }^{4}$.

\section{Tant et tant de chevaux}

Dans son roman Tant et tant de chevaux, Luis Ruffato propose un portrait de São Paulo et une réflexion sur la formation de l'espace urbain de la mégalopole brésilienne. Dans un texte de 2010, Dominique Stoenesco informe que Ruffato demeure peu connu du public brésilien comme aussi du public français. Ruffato, qui a été vendeur de popcorn, a suivi une formation de mécanicien tourneur pendant laquelle il a fréquenté la 
bibliothèque de son école et $\mathrm{y}$ a découvert la littérature mondiale qui l'entrainait au-delà de ses frontières régionales.

La ville représentée dans le roman de Ruffato semble témoigner du destin collectif d'un corps social anéanti par l'enchevêtrement des forces de pouvoir. Comme le dit Stoenesco (2010, p. 275), la problématique abordée dans ce roman n'est pas de façon explicite la question des modalités de représentation de l'histoire, et encore moins la question des mythes identitaires brésiliens, mais plutôt la question d'une mémoire collective déboussolée. Comme les chevaux du titre, les mots doivent galoper à grande vitesse à travers 69 tableaux numérotés, dans un langage hétérogène et hybridisé, où s'entremêlent mots d'argot, mots étrangers et formes inventées. 69 textes exposent des dialogues, ou micro-récits autonomes, qui se déploient en une seule journée, dans un langage fragmenté, tout en formant un tableau impressionnant de São Paulo, où déambulent des laissés-pour-compte, de très riches et des messieurs-tout-le-monde, comme des troupeaux abandonnés.

\section{Fonder la ville à travers l'écriture}

Dans Ce qu'il reste de moi, nous avons déjà dit que Laurel est le guide de la ville, car il entend écrire la fondation de Montréal par Paul Chomedey et Jeanne Mance. Pour lui, la ville est un être vivant qui grandit, change, se modifie en fonction des événements historiques, sociaux, politiques et culturels depuis sa fondation, avec une mémoire qui doit être connue de ses nouveaux habitants. Cette quête des origines est toutefois critiquée par son père, Thomas, un cinéaste de renom, qui ne peut pas comprendre pourquoi on veut écrire sur des faits historiques, dans un siècle déjà dominé par une haute technologie.

- Pardonne-moi, je t'ai perdu. Le cœur de qui?... De quoi parles-tu?....

- Jeanne Mance!... le cœur de Jeanne Mance!

- Tu veux écrire un livre sur Jeanne Mance!

- Pas un livre SUR mais, comme je l'expliquais, inspiré DE, nourri par cet élan de mysticisme total qui a donné naissance à... tout ça, à ici, à Montréal, bref, tu comprends...

Thomas n'en revient pas. On est bien au XXìme siècle, on transplante des cerveaux transgéniques et des cœurs en plutonium, on nage dans les nanotechnologies, on flirte avec les cyborgs, on se déplacera incessamment par ondes virtuelles et voilà que ce surdoué apathique, cet ersatz de lui-même s'apprête à écrire un livre sur la 
cofondatrice de Montréal au temps des trois-mâts et du scorbut - un projet tellement passionnant qu'il sent qu'il pourrait de suite être projeté dans un sommeil éternel!

- Formidable, répète-t-il faiblement (PROULX, 2015, p. 150).

La stupéfaction et l'ironie de Thomas révèlent une sorte de pensée pragmatique qui considère que le passé historique, temps des «trois-mâts et du scorbut», importe peu dans une époque de développement scientifique. C'est exactement ce manque d'esprit diachronique, qui refuse tout lien du présent avec le passé historique, apte à former le réseau mnésique de la ville, qui incite Laurel à poursuivre son projet. Celui-ci se promène avec les cendres de sa grand-mère, persuadé que le portrait actuel de la ville multiculturelle trouve ses origines dans les cendres des ancêtres qui ont bâti Ville-Marie. Son rôle d'écrivain, qui revisite l'histoire de la ville, ramène à des réflexions sur les relations entre les Jésuites et les Amérindiens (vus comme des Sauvages), les premières formes de cultures alimentaires pour sédentariser les colons (blé d'Inde, courges et tournesol), la médecine douce avec les fruits délicats (les gadelles, les aubépines, les pourpiers sauvages, l'ail des bois) (PROULX, 2015, p. 82).

En plus de la description de la configuration de cette cité idéale, Laurel développe également une réflexion sur le sens de l'histoire et sur la difficulté de sa représentation, lorsqu'il aborde l'acculturation forcée des dits Sauvages et les stratégies militaires de Paul de Chomedey, sieur de Maisonneuve, sur le plan de la moralité, pour bien administrer Ville-Marie : menace de bannissement ou de prison pour usage d'alcool, blasphèmes, jeux de hasard, adultère et inconduites avec les Sauvagesses... (PROULX, 2015, p. 84). Naturellement, l'écrivain exhibe, comme des mises en abyme, les hypotextes historiques montrant le voyage de Paul de Chomedey et Jeanne Mance des côtes de La Rochelle jusqu'à la Nouvelle-France, au cœur de l'Isle de Montréal, «une cité qui parviendrait à s'extraire du temporel pour se consacrer au divin» (PROULX, 2015, p. 10). Au cœur de la sauvagerie informe, Jeanne Mance fonde un hôpital, où elle sert comme infirmière, avec sa vision de rêve d' "un paradis terrestre touffu, des chênes, des hêtres, des cèdres, des peupliers...» (PROULX, 2015, p. 11). Le croisement du texte historique et du récit fictionnel vient refléter ainsi le résumé d'une traversée du passé impliquant des changements dans les références des archives historiques, dans la mesure où l'écrivain 
permet une actualisation successive des aspects différenciés de l'expérience de fonder une cité. La matérialisation explicite de l'espace-charnière de l'écriture de Laurel montre que la problématique abordée concerne moins le besoin de montrer la dénotation du fait historique et la perpétuation des mythes fondateurs nationaux, mais plutôt la question de l'actualisation d'une mémoire collective plurielle dès sa naissance. Laurel ne veut pas non plus saisir la configuration de la métropole québécoise comme sémiosphère de l'usage de la haute technologie, comme le pense son père, mais interroger le passé historique (la zone mixte, comme espace-charnière, de la Folle Entreprise de Jeanne Mance) pour pouvoir expliquer le temps présent d'un espace-charnière apte à faciliter la convivialité de plusieurs peuples.

\section{L'espace-charnière de Ruffato}

Stoenesco (2010, p. 274) montre que Ruffato est d'une génération des intrigues délinéarisées, et qu'il s'est inspiré des visions réalistes de Faulkner, Joyce et Machado de Assis qui matérialisaient déjà au $\mathrm{XIX}^{\text {ème }}$ siècle et au $\mathrm{XX}^{\text {ème }}$ siècle le métalangage dans leurs romans pour mettre en valeur le rôle des écrivains. Dans Tant et tant de chevaux, chaque élément de la mosaïque trouve une écriture propre, chaque tableau trouve une langue pour évoquer les thèmes révélateurs d'une société proche d'un état de décomposition, sujette à la violence urbaine. Ruffato utilise une grande variété de langages (régionalismes, archaïsmes, néologismes, mots étrangers, mots d'argot), car pour l'auteur le rapport au réel ne peut pas être envisagé en dehors du langage, ce qui est le propre de l'espace-charnière.

Luiz Ruffato considère que les expressions de discrimination sociale découlent d'un usage de la langue révélant les relations de pouvoir qui définissent la position des interlocuteurs. La langue est également un élément identitaire, un marqueur social et culturel que l'écrivain propose. Dans la structure de narration proposée, il compose des portraits minutieux indiquant des comportements vus sous une grande variété d'angles. Il tisse aussi des relations complexes entre ces personnages qui agissent comme un troupeau égaré, comme s'il était un berger s'efforçant de rassembler son troupeau de brebis dans les labyrinthes de cette grande sémiosphère «paulista ${ }^{5} »$. 
Ruffato a toujours affirmé que la structure de son roman était une sorte d'atelier littéraire. Il a fait ainsi une expérimentation littéraire, empruntant des techniques au montage filmique et aux arts plastiques pour composer la réalité multiforme de São Paulo. Ainsi, le travail de l'écrivain ne serait pas celui d'un reporter prêt à décortiquer le terrain social de la ville, mais celui de quelqu'un qui fusionnerait source d'information et objet esthétique. Remarquons l'articulation langagière dans ce passage qui parle de personnages désemparés dont les bornes de frontières sont tout à fait brouillées :

(...) et rien rien de tout cela ne restera rien le quartier se transformera en terrain vague sous chaque réverbère éteint à chaque coin de rue bistros rideau de fer à demi baissé chaque bicoque chaque demeure tour chaque chat chien chaque sac d'ordures et tout aura été vain são paulo entière décadence et tous l'abandonneront et une ville fantôme comme celle des westerns en noir et blanc d'un vidéo-club qu'il regardait assis sur son lit en mangeant des pop-corn grillés au micro-ondes et en buvant du

coca-cola

surgira

pourquoi

tout

si d'ici quelques milliers d'années la terre finira dans une hécatombe elle cessera de tourner froide inerte... (RUFFATO, 2005, p. 75).

Le fragment de texte présente plusieurs effets visuels advenant dans la langue (motvalise, invention de mots, allitérations, répétitions de la même phrase) qui font ressortir deux sortes de pensée : celle relative à la vie hallucinée et agitée des personnes de la grande ville et celle de la difficulté qu'elles ont pour s'exprimer du fait qu'elles sont immergées dans un monde apocalyptique. Ces personnages se trouvent « asémiotisés » de telle sorte qu'ils ne sont pas du tout capables de surmonter leur situation de néant (soumis à toute sorte de vols, de viols, de hold-up) pour resémiosphériser leur espace social. Ils représentent une série d'exilés, de Noirs, d'immigrants portugais pauvres, d'immigrants d'autres régions plus pauvres du Brésil, des personnes sans identité, dont les repères sociaux sont perdus, écrasés dans la grande ville qui devient alors le personnage le plus important du roman.

Pour revenir à Ce qu'il reste de moi, remarquons que la tante de Laurel, Gaby, enseigne le français à des immigrants, ce qui pose aussi un problème de l'écriture de l'histoire de la ville, à travers un procédé narratif de métalangage: 
Yuli, Samantha, Yin, Jaimal, Barbara, Constant, Mark, Pino, Lily, Jesus, Assouf, Pakir, Driss, Mohamad, Tonio, Maria, Redostin, Laila... Quand ils sont assis devant elle, leurs visages de moins de vingt ans et de plus de cinquante ans sont uniformément rajeunis par une confiance absolue. Quand elle est debout devant eux, à disséquer le mystère des participes passés pronominaux ou celui de l'accord des adjectifs numéraux, ou à jouer les mentors sardoniques comme aujourd'hui, elle est celle qui compte le plus dans leur vie, elle est leur plus-que-mère, elle est leur porte d'entrée sur le nouveau monde. Il n'y a pas de mot pour décrire parfaitement cette relation éphémère et intense, comme il n'y a pas de justification au fait qu'une mère se mette à aimer un enfant plus que les autres, à trembler davantage pour Laila que pour Lily, par exemple (PROULX, 2015, p. 213).

Dans l'immersion française de Gaby, il y a aussi des effets visuels des différences entre les élèves: des musulmanes avec ou sans foulard, des Africaines excisées, des Roumaines exploitées par des souteneurs, de petites Coréennes épuisées par les mauvais boulots, des Sud-Américaines poursuivies par l'immigration, des Indiens de la caste des brahmanes, de vieux Haïtiens. C'est une expérience de multiplicité culturelle qui s'installe peu à peu dans les salles de cours, vues comme des espaces-charnière, comme lieu de passage où l'on circule d'une langue à une autre, mais aussi où on commence à apprendre à s'unir autour d'un besoin commun, celui de maitriser les codes socioculturels de la sémiosphère d'accueil. Justement, la pensée d'un espace urbain comme un lieu de sémiosphère a l'avantage de montrer les interrelations entre signes culturels qui contribuent aux interactions entre l'individuel et le collectif dans une plateforme de réseautage. Ici aussi il y a une monstration visuelle des usages de la langue, dans le but de faire ressortir le caractère discontinu et progressif de l'acculturation (sémiotisation) des immigrants.

Aujourd'hui, on célèbre la lettre P.... Pakir et Pino, par pitié, ne papotez plus... Des fruits?... Patate, bouh, c'est une légume, ça Barbara, papaya, excellent, pomme, bien sûr, pamplemousse, bravo, Tonio, pinapel, Lily?... Tu veux dire ananas... Prune, poire, pastèque, pruneau... (PROULX, 2015, p. 213).

... Des métiers maintenant... Ppp... Plombier, oui! Papa, ah, ah! Policier, hélas, oui. Professeur! À qui le dites-vous? et dur métier à part ça. Photographe, très, très bien. Pape! Pourquoi pas? J'adore ça, pape, et le féminin est ? Papesse !... Pharaon?... Excellent!... Qui a dit Pharaon? (PROULX, 2015, p. 215). 
À travers ces bribes de mots et de phrases, ces allitérations avec la lettre P, Proulx nous montre comment ils font l'apprentissage de la langue, ces êtres qui circulent dans la périphérie de la sémiosphère urbaine, réalisant des efforts considérables pour passer au centre, afin d'y occuper des positions énonciatrices déterminantes.

Si l'on compare les deux romans, il y a lieu de noter que, dans Tant et tant de chevaux, les ruptures langagières dénotent l'idée d'une ville dévastée qui met en déroute toute tentative pour réaliser, à travers elle, une configuration répondant aux exigences de l'intelligibilité et de l'esthétique. L'espace charnière (matérialisé dans les rues, les églises, les supermarchés, dont les frontières sont en train d'être repoussées par la dynamique sémiosique des nouveaux venus qui peut bouleverser la stabilité du Centre) y fonctionne comme passerelle pour le règne de la contingence et du hasard, la défaite de tout sens. Toutefois, le roman de Proulx va dans le sens inverse, tout en signalant qu'une configuration ordonnée est possible par l'entremise des passerelles des charnières exposées (places, églises, écoles); il est possible de regrouper toutes les identités éclatées, qui se trouvent désémiosphérisées, autour d'un projet de convivialité désirée dès la fondation de la ville.

\section{Les espaces gigognes, en guise de conclusion incomplète}

Nous avons déjà éclairé le fait que le mot «gigogne» définit un emboîtement d'objets. Par conséquent, un espace-gigogne serait celui qui contiendrait les propriétés d'autres espaces qui s'y dissimuleraient ; un horizon qui abrite d'autres horizons. Ceci est d'une importance primordiale pour l'étude des espaces urbains en concurrence dans les sémiosphères, parce que ce qui est signalé concerne une incorporation et une transformation d'espaces dans le même territoire.

Dans le roman brésilien, São Paulo, par sa croissance démesurée et imprévisible, parle comme ville, comme centre même de la grande sémiosphère brésilienne, tout en arborant ses collages parataxiques, fruits de la difficile intégration entre Noirs, Indiens, Blancs, Nordestins, pauvres, classes moyennes ou supérieures, enfin tous ces personnages d'origines et de conditions sociales les plus diverses. On y trouve une multitude de langages et des valeurs qui s'emboîtent les uns dans les autres : ils sont les témoins d'une société victime d'une violence incontrôlée, du chômage, du stress, de l'endettement 
des classes moyennes et des croyances religieuses fondamentalistes. La violence, une présence marquante dans les tableaux et dans les dialogues, est le dénominateur commun de discussion des millions d'habitants de São Paulo. Les collages et les assemblages, une fois mis en relation par les charnières, qui confondent les frontières, émergent pour agencer une multiplicité d'images ou de fragments de langages en une séquence unique qui peut fonctionner comme espace gigogne. L'assemblage d'éléments hétéroclites permet l'insertion de textes non-fictionnels (petites annonces, horoscopes, faits divers, etc.) dans le discours fictionnel tout en autorisant la formation de plusieurs gigognes. Comme le dit encore Stoenesco (2010), «ces collages rassemblent dans la simultanéité, ce que la littérature traditionnelle déploie dans la succession».

Proulx produit également des gigognes, un «gisement mystique», avec des vécus différenciés: la synagogue où va l'hassid, le quartier des spectacles où l'artiste donne une performance, le Centre Bell où la foule crie, galvanisée par un but des Canadiens, plusieurs histoires de vie enfouies sous les artères de Montréal, depuis que Jeanne Mance est venue il y a plus de quatre cent ans et y a laissé son cœur. Il y a de la diffraction ici aussi, avec la force d'un projet esthétique associé à l'ouvrage et à sa construction. Pourtant, la trame historique de Laurel vient assurer une chaîne de sens, et indiquer que le rêve utopique d'une société « mixte », qui existe depuis la fondation de Montréal, peut être capable de réunir des peuples différenciés dans cette sémiosphère urbaine.

$\mathrm{Au}$ fond, la présentation narrative des deux romans suit un cheminement similaire. On transite du contact à l'emmagasinement des références, des charnières aux gigognes. La narrativité se fait transmission, les jeux de voix et les métissages des langages parviennent à faire découvrir une réalité complexe, qui s'ouvre sur l'interculturel, mais exhibe les effets d'une discrimination envers les migrants, encore présente dans les sociétés visées. São Paulo, devenue la locomotive du Brésil grâce à la sueur des travailleurs immigrés, continue d'affronter des problèmes d'exclusion sociale. Quelques centaines d'années plus tard, Ville-Marie - devenue Montréal - abrite des hommes et des femmes qui se battent, chacun à leur façon, pour redresser les fortifications d'une vie en proie à diverses vicissitudes, mais rencontre également ces problèmes d'exclusion/ inclusion. C'est ainsi que Gaby, qui reçoit la visite de Laurel dans sa classe de français, 
s'exclame: «Pas question qu'il s'empare de l'identité éclatante de ses élèves et la dégrade dans les personnages de fiction» (PROULX, 2015, p. 215).

Cette scène s'avère exemplaire, pour synthétiser cette problématique de l'existence des sémiosphères dotées de plusieurs chronotopes en concurrence, car Gaby témoigne qu'elle croit aux richesses foisonnantes des cultures immigrantes. Elle se méfie toutefois des frontières créées par les langages de fiction, supports de plusieurs couches de sens. En ce moment, elle a besoin de se bâtir un monde d'intégration multiforme, et souhaite saisir une clôture significative qui lui permette d'accomplir son rêve de la Folle Entreprise.

\section{Références}

FORTIER, Frances, MERCIER, Andrée (Dir.). La transmission narrative : Modalités du pacte romanesque contemporain. Montréal: Éditions Nota Bene, 2011.

GERVAIS, Bertrand. La ligne brisée, Labyrinthe, oubli et violence. Logiques de l'imaginaire - tome II. Montréal: Le Quartanier, 2008.

IMBERT, Patrick. Trajectoires culturelles transaméricaines. Ottawa: Presses de l'Université d'Ottawa, 2004.

. Transculturalité et transdisciplinarité: le Canada et les Amériques. Interfaces Brasil/Canadá, v. 11, n.1, 2011, p. 9-27.

LOTMAN, Youri. La sémiosphère. Limoges: PULIM, 1999.

OUELLET, Pierre. L'esprit migrateur, Montréal: Trait d'Union, 2003.

PROULX, Monique. Ce qu'il reste de moi. Montréal: Boréal, 2015.

RUFFATO, Luiz. Tant et tant de chevaux. Trad. Jacques Thiériot. Paris: Éd. Métaillé, 2005.

SOUZA, Licia Soares de. Figures spatiales de Montréal dans une perspective de géopoétique urbaine. Interfaces Brasil/Canadá, v. 15, n. 2, 2015, p. $42-67$.

STOENESCO, Dominique. Histoires individuelles et histoire collective, esthétique et éthique dans Eles eram muitos cavalos de Luiz Rufato. In: OLIVIERI-GODET, Rita (Dir.), Écriture et identités dans la nouvelle fiction romanesque. Rennes: Presses Universitaires de Rennes, 2010, p. 269-281.

Liens sur le film La Folle Entreprise, sur les pas de Jeanne Mance https://jeannemancefilm.wordpress.com/notes-biographiques/ https://jeannemancefilm.wordpress.com/ http://comite.maisonneuve.free.fr/visitedannabelloyola.html http://montreal.ctvnews.ca/annabel-loyola-speaks-about-jeanne-mance-1.835586 http://www.cfqlmc.org/bulletin-memoires-vives/derniere-parution/583 


\section{Notes}

${ }^{1}$ Departamento de Estudos Literários, Universidade do Estado da Bahia, Salvador, Bahia, Brasil. Pesquisadora do Cnpq. liciasos@hotmail.com.

${ }^{2}$ Y. Lotman (1999) ne propose pas un modèle d'abstraction des phénomènes culturels, mais un processus de modélisation de la « réalité sémiotique » qui les sous-tend. La sémiosphère est l'ensemble de références d'une culture donnée, nécessaire à l'instauration des systèmes sémiotiques qui la composent. Elle est en interaction permanente avec ses parties. La sémiosphère est une expérience sémiotique collective qui se traduit par «présomption de sémioticité ». Elle précède et en même temps rend possible toute sémiosis en vigueur dans le cadre d'échanges culturels. Elle est l'unité de base de la signifiance humaine elle-même. Hors cet espace de référence, il ne peut y avoir ni communication ni signification. La sémiosphère se définit par plusieurs caractéristiques: la binarité, l'asymétrie, la panchronie, l’hétérogénéité, la transitivité.

3 Voir: Figures spatiales de Montréal dans une perspective de géopoétique urbaine (2015).

${ }^{4}$ D'après la réalisatrice du film La Folle Entreprise, sur les pas de Jeanne Mance, Annabel Loyola, « Jeanne Mance n'a pas laissé d'écrits autobiographiques et les incendies ont effacé une grande partie de sa mémoire de part et d'autre de l'Atlantique. Tout ce que nous savons c'est grâce aux registres, aux actes notariés et surtout grâce à ses contemporains qui ont laissé des écrits d'une valeur inestimable pour notre histoire. Ce sont ces documents à la source qui m'ont inspirée dans la réalisation du film et qui parfois, et par chance pour moi qui suis non-historienne, connaissaient des éditions critiques. » Accessible sur : <http://www. cfqlmc.org/bulletin-memoires-vives/derniere-parution/583>.

5 Qui est relatif à la ville de São Paulo. 